\title{
Rare variation in the origin of the right vertebral artery
}

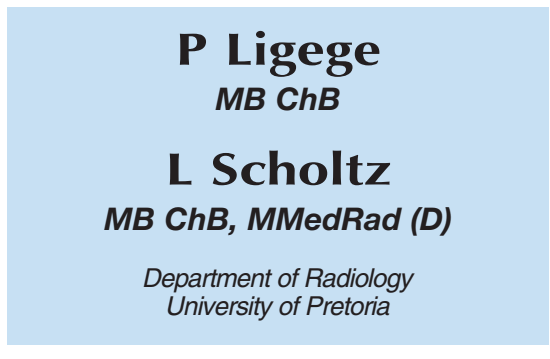

\section{Introduction}

Anomalous origin of the right vertebral artery from the distal part of the aortic arch is very rare and is discovered as an incidental finding during an angiographic study or in laboratory specimens. It has been suggested that these anomalies are of diagnostic importance and may be associated with arteriovenous malformations or cerebral aneurysms.

Although no conclusive evidence exists that vertebral artery anomalies are associated with a higher incidence of cerebrovascular disorders, some authors hypothesise that the anomalous origin and distribution of the large aortic arch vessels can cause changes in cerebral haemodynamics that may lead to cerebrovascular anomalies.

\section{Case report}

A 40-year-old man was referred to the emergency unit with a clinical picture and signs suggestive of a cerebrovascular accident. No medical history was available and all attempts to contact the family failed.

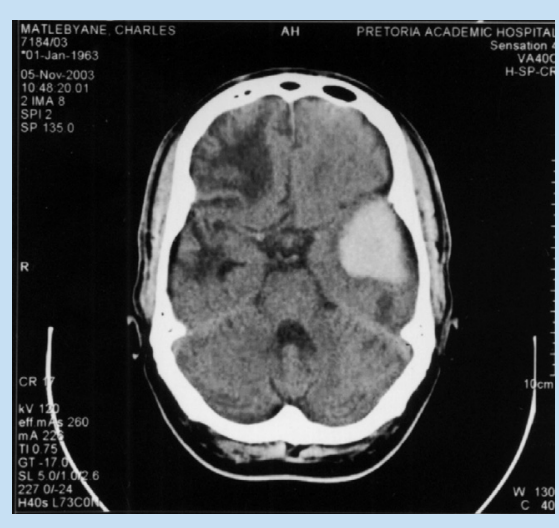

Fig. 1. CT scan of the patient depicting a large intra-cerebral haematoma in the left temporal region with area of gliosis in the right frontal and temporal area.

A pre-contrast CT examination showed a $3 \times 3 \mathrm{~cm}$ haemorrhage that extended from the left temporal lobe superiorly to the level of the third ventricle with surrounding oedema (Fig. 1). There was no evidence of intraventricular breakthrough or subarachnoid haemorrhage. The midline was displaced $3 \mathrm{~mm}$ to the right. A large low-density lesion situated in the ter-
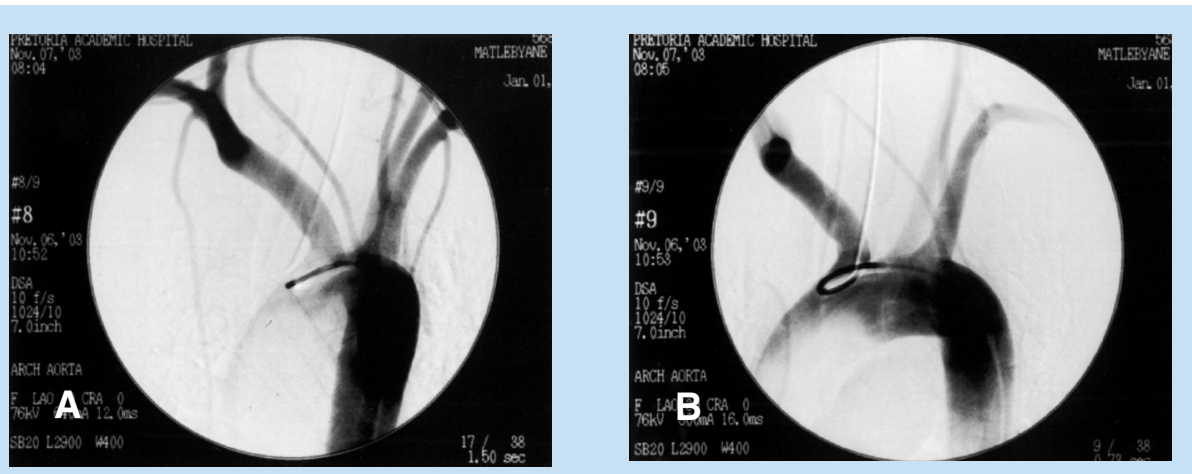

Figs $2 \mathrm{a}$ and $b$. AP and left anterior oblique projections during angiography showing the origin of the right vertebral artery distal to the left subclavian artery.

ritory of the right middle cerebral artery was also present with ex vacuo enlargement of the ipsilateral ventricle in keeping with an old infarct.

For further evaluation of the cause of the intracerebral haemorrhage, four-vessel digital subtraction angiography was embarked on 10 days after presentation. During the procedure some difficulty was experienced with the selective catheterisation of the supra aortic vessels with a headhunter, largely because of the repeated catheterisation of a vessel that originated from the arch but seemed to cross the midline from left to right.

It was then decided to perform an aortic arch flush to try and establish the anatomy of the arch and its branches, using a pigtail catheter. The right vertebral artery was found not to arise from the right subclavian artery, but instead its origin was distal to the left subclavian artery as the last of the supra-aortic vessels (Figs 2a and b).

No arteriovascular malformation or aneurysm could be demonstrated.

\section{Discussion}

Many variations occur in the number and position of the vessels that arise from the aortic arch. A common form with three branches arising from$$
\text { . }
$$ .}




\section{CASE REPORT}

the aortic arch is found in approximately $80 \%$ of persons. The branching order is right brachiocephalic artery, left common carotid artery and left subclavian artery.

When more than three branches arise from the aortic arch, the vertebral arteries are usually added. A common form, with four vessels, is one in which the left vertebral artery arises between the left common carotid artery and the left subclavian artery. The frequency of this variant ranges from about $2.4 \%$ to $5.8 \%$.

A very rare variation is the origin of the right vertebral artery distal to the left subclavian artery, with only eight cases previously published in the literature. The reported order of branching has then been as follows: right brachiocephalic artery, left common carotid artery, left subclavian artery and finally the right vertebral artery.

The right vertebral artery usually arises: (i) from the upper, posterior surface of the subclavian artery (80\%); (ii) directly from the aortic arch (5\%); (iii) from the right common carotid artery $(0.28 \%)$; or (iv) from the site of bifurcation of the brachiocephalic trunk into the right subclavian artery and right common carotid artery (1.11\%).

A detailed knowledge of the anomalous origins of supraaortic vessels is of importance in patients who have to undergo four-vessel angiography. If a vertebral artery cannot be found in the usual position, the possibility of such a variant must be considered. The exsistence of these anomalies also accounts for the policy in many practises, of routinely performing an angiogram of the aortic arch before attempting selective catherisation of the carotid and vertebral arteries.

\section{Further reading}

1. Bergman RA, Thompson SA, Adel KAfifi, et al. Compendium of human anatomic variation. Text, Atlas, and World Literature 1989; 60-63, 359.

2. Lemke A-J, Benndorf G, Liebig T, et al. Anomalous origin of the right vertebral artery: Review of the literature and case report of right vertebral artery origin distal to the left subclavian artery. American Journal of Neuroradiology 1999; 20: 13181321.

3. Bergman RA, Adel K Afifi, Miyauchi R, et al. Vertebral arteries. In: University of Iowa Health Care, Virtual Hospital, ed. Illustrated Encyclopedia of Human Anatomic Variation: Opus II: Cardiovascular System: Arteries: Head, Neck and Thorax 